\title{
Masculinidad y relaciones sexuales entre humanos y animales en contextos agroganaderos andaluces
}

\author{
Masculinity and sexual relations between humans and animals in \\ Andalusian livestock farming
}

\author{
Coca-Pérez, Agustín \\ Universidad Pablo de Olavide \\ acocper@upo.es \\ https://orcid.org/0000-0001-6377-1898 \\ Cáceres Feria, Rafael \\ Universidad Pablo de Olavide \\ rcacfer@upo.es \\ https://orcid.org/0000-0002-9471-1211 \\ Valcuende del Río, José María \\ Universidad Pablo de Olavide \\ jmvalrio@upo.es \\ https://orcid.org/0000-0003-1161-2611
}




\section{Resumen}

La sexualidad entre humanos y animales no puede ser entendida exclusivamente como una categoría identitaria, una desviación o patología, ni tampoco como la expresión de una sexualidad descontrolada. Hasta ahora en el estudio de la sexualidad humano animal han predominado las visiones médico-psiquiátricas. Sin embargo, las investigaciones en Antropología ponen de manifiesto la necesidad de estudiar los significados de estas prácticas sexuales en función de los contextos locales. En el caso analizado en el sur de España las relaciones sexuales con animales de algunos varones están enmarcadas en un proceso de aprendizaje de la sexualidad, indisociablemente vinculado con la construcción de la masculinidad.

Palabras clave: España; Andalucía; antropología; sexualidad; masculinidad; zoofilia.

Abstract

Sexuality between humans and animals cannot be understood solely as an identity category, a deviation, or pathology, nor as the expression of unbridled sexuality. So far, medical and psychiatric perspectives have dominated the study of human-animal sexuality. However, research in Anthropology highlights the need to study the meanings of these sexual practices according to local contexts. In the case analysed here, in southern Spain, sexual relations maintained with animals by some men are framed within a process of sexual apprenticeship, intrinsically linked with the construction of masculinity.

Key words: Spain; Andalucia; anthropology; masculinity; sexuality; zoophilia. 


\section{Introducción}

La presencia de los animales ha sido una constante en nuestras vidas, como lo ha sido también en los lugares en los que hemos desarrollado una buena parte de nuestras investigaciones. Los animales eran para la gente con la que aprendíamos investigando: herramientas, mascotas, medios de transporte, recursos, símbolos... Pero los animales eran algo más, también estaban presentes en los juegos de los niños y en los procesos de aprendizaje de la sexualidad de los varones. Una realidad oculta pero conocida, que todo el mundo miraba, pero no veía. Nosotros también sabíamos de estas prácticas sexuales, ahora bien, nunca fueron abordadas en nuestras investigaciones. Lo serán mucho tiempo después cuando incorporemos nuevas preocupaciones de investigación aparentemente divergentes: la sexualidad y las relaciones socioambientales. Nuevas perspectivas nos permitieron dar valor a una información que anteriormente consideramos secundaria. No sólo fuimos nosotros los que, a pesar de conocer esta realidad, la obviamos, hay temas de investigación que constituyen un auténtico tabú (Cáceres y Valcuende, 2020). No por casualidad nos encontramos ante uno de los primeros trabajos antropológicos que aborda directamente las relaciones sexuales entre humanos y no humanos en España ${ }^{1}$.

Este renovado interés por analizar las relaciones entre animales y humanos nos permitió volver a nuestros cuadernos de campo y recuperar toda una serie de anotaciones que, hasta hace poco tiempo, habíamos considerado anecdóticas. El cúmulo de evidencias acopiadas durante años, reinterpretadas desde la distancia, nos posibilitó mirar de otra forma una realidad no reconocida. A partir de ahí, nos pusimos a trabajar buscando nuevos testimonios de hombres que habían tenido contactos sexuales con animales o que conocían dichas prácticas en el sur de España. Fue una tarea complicada ya que actualmente estas relaciones están siendo resignificadas y cada vez es más difícil obtener información por el estigma que pesa sobre las mismas. Unos prejuicios que, dicho sea de paso, se ponen en evidencia en una buena parte de las investigaciones que abordan esta temática. Pero nos dimos cuenta también de la centralidad de la etnografía a la hora de aproximarnos a la realidad cotidiana de la sexualidad. Sólo a través de investigaciones de largo recorrido, que permitan un conocimiento profundo de las sociedades observadas y un cierto nivel de confianza entre los investigadores y los informantes nos pueden permitir intentar algo que en cierta medida es una quimera, y que sólo se puede conseguir parcialmente: comprender el mundo como si fuéramos "el otro" ¿Pero ¿qué buscamos con esta aproximación? Fundamentalmente analizar la significación de la sexualidad con animales para los hombres en los contextos rurales campesinos.

Partimos de que las prácticas sexuales con animales de los varones en el ámbito rural andaluz están enmarcadas en un proceso de aprendizaje de la sexualidad, indisociablemente vinculado con la construcción de la masculinidad. A partir de este estudio exploratorio cuestionamos las visiones que plantean exclusivamente la zoofilia desde perspectivas patologizantes. Desde nuestro punto de vista, la comprensión de las prácticas sexuales con animales no puede desvincularse de la sexualidad normativa $\mathrm{y}$ de lo que se ha venido en denominar masculinidad hegemónica (Connell $\mathrm{y}$ Messerschmidt, 2021). En este trabajo nos aproximamos precisamente a las normas que rigen las relaciones sexuales entre los hombres y los animales, y a la significación de estas prácticas a lo largo del ciclo vital de los hombres, que mantienen "un secreto" a través del cual refuerzan la solidaridad del grupo de iguales, con el que aprenden el significado de la masculinidad (Valcuende, 2003).

\footnotetext{
${ }^{1}$ El primer trabajo que aborda esta temática fue realizado por los mismos autores, ver Coca, Valcuende y Cáceres (2019). En este texto profundizamos y ampliamos el trabajo previo publicado en la revista Sexualties. Pensábamos que era fundamental dar a conocer este trabajo, facilitando su lectura en castellano y contribuir así a la devolución de resultados en el contexto en el que se realizó la investigación.
}

Revista del Laboratorio Iberoamericano para el Estudio https://doi.org/10.46661/relies.6386

Sociohistórico de las Sexualidades 


\section{Del bestialismo a la zoofilia}

Las relaciones sexuales entre humanos y animales han sido utilizadas para estigmatizar a los "otros". Desde las sociedades urbanas, el bestialismo se sitúa en el ámbito rural, en los grupos subalternos o en "sociedades primitivas". Kinsey (1948) localizaba los casos de sexualidad humano animal en Estados Unidos entre jóvenes granjeros. Para Miletski (2002), el estereotipo de zoofílico es el del campesino pobre e ignorante. Las alusiones a este tipo de sexualidad son especialmente profusas en contextos de colonización (Amodio 2012; Bazant, 2002; Vega 1994). Esto no es casual ya que la acusación de prácticas sexuales consideradas inmorales era una clara forma de justificar el control y dominio sobre los bárbaros, los indígenas, los subalternos... El bestialismo, desde el discurso del poder, aúna al humano y a la bestia. Ambos deben ser enseñados, dominados, castigados, en términos de Foucault (1975), disciplinados a través del control de sus cuerpos y de su sexualidad. Desde estos discursos, las sociedades "civilizadas" y urbanas estarían libres de esta lacra. Sin embargo, cuando comienzan a visualizarse los casos de relaciones sexuales de humanos con animales en las ciudades cambia la perspectiva; deja de ser un problema moral para convertirse en una cuestión médica. Lo que para los "primitivos" es inmoralidad para los civilizados es trastorno mental.

Los motivos de reprobación de este tipo de sexualidad no son los mismos en todas las sociedades ni se han mantenido invariables en el tiempo. En Europa, hasta finales del siglo XIX, se hablaba de sodomía y bestialismo; conceptos que tenían un claro componente moral y que penalizaban estas conductas desde el punto de vista religioso y también legal, en contextos en que ambas esferas eran indisociables. En 1886 el psiquiatra alemán Kraft-Ebing (1894) acuña los conceptos zooerastia y zoofilia para referirse a una conducta patológica que implica la atracción sexual y emocional por los animales, y reserva el vocablo bestialismo para las prácticas instrumentales encaminadas exclusivamente a satisfacer el deseo sexual. Las relaciones sexuales entre humanos y animales dejan de ser solo conductas reprobables moralmente para ser calificadas como patología. Havelock Ellis (1923) siguiendo esta distinción considera que el bestialismo es practicado por individuos de bajo nivel cultural mientras que la zoofilia se da entre personas de las clases cultas con trastornos mentales. A diferencia del bestialismo, la zoofilia ya no sólo nomina las prácticas sino también a aquellos que la realizan ("zoofílicos"). La "perversión" o la "inmoralidad" se desplaza del acto a la propia persona. Un proceso que no sólo se produce en este tipo de comportamientos, se da, igualmente, en otras sexualidades no reproductivas (relaciones entre personas del mismo sexo, masturbación, fetichismo...) (Foucault 1976; Rubin 1984).

La distinción entre zoofilia y bestialismo se ha mantenido dentro de la psicología y la psiquiatría. La zoofilia aparece recogida en el Diagnostic and Statistical Manual of Mental Disorders (DSM-5 ed.) de la American Psychiatric Association (APA) (2013) como una parafilia. Con esta visión de las relaciones sexuales humano animales no resulta extraño que la literatura científica sobre el tema tenga fundamentalmente una orientación médico/psiquiátrica. Encontramos, por un lado, aquellos trabajos que abordan esta sexualidad desde un punto de vista clínico-terapéutico (Cerrone 1991; Álvarez and Freinhar 1991; Beetz 2002; Miletski 2001; Peretti and Rowan 1982; Earls and Lalumiere 2009); y, por otra parte, los que la interpretan desde una perspectiva forense-criminológica ya que frecuentemente se relacionan estas prácticas con delitos de violencia (Aggrawal 2011; Ascione 2005; Duffield, Hassiotis and Vizard.,1998; Flynn 1999; Hensley, Tallichet and Singer 2006).

Aún más exiguas son las investigaciones que abordan la zoofilia desde las ciencias sociales. Por su repercusión destaca el estudio de Dekkers (1994) en el que analiza las relaciones sexuales con animales en la psicología, el derecho, la literatura, el arte o la publicidad. También algunas 
investigaciones se han ocupado del bestialismo, sobre todo, a partir del análisis de los procesos judiciales contra acusados de estas prácticas (Liliequist 1991; Rydström 2003; Salisbury 1991).

Esta visión medicalizada y la dificultad metodológica de acceder a personas que tienen relaciones sexuales con animales son las principales razones que explican el desinterés de la sociología por este tema. Sin embargo, esto ha comenzado a cambiar con la visibilización de estas prácticas sexuales en internet. Las redes sociales virtuales se han convertido en espacios de confluencia y reivindicación de esta sexualidad (Williams and Weinberg 2003; Kavanaugh and Maratea 2016). Las personas que la practican le dan una interpretación positiva, autodenominándose zoosexuales o simplemente zoos, y creando sus propias comunidades, "zoo community" (Durkin, Forsyth and Quinn 2006). La articulación de estas comunidades de zoosexuales a través de internet ha facilitado el acceso de los investigadores a esta realidad (Jenkins and Thomas 2004; Maratea 2011; Kavanaugh and Maratea 2016; Williams and Weinberg 2003). Es en este contexto en el que algunos investigadores comienzan a hablar de la zoofilia como una orientación sexual (Miletski 2017).

La antropología no constituye una excepción, las investigaciones antropológicas sobre relaciones sexuales entre animales y humanos son escasas (Davis and Whitten 1987). Los estudios etnográficos no van más allá de estas escuetas referencias (Beidelman 1961; Chaplin 1963; Delaney 1991; EvansPritchard 1956; Malinowski 1975, Williams 1966). Entre los trabajos que se ocupan del bestialismo de forma más precisa está el de Devereux (1948) sobre los mohaves de Norteamérica y el de LeVine (1959) sobre los gusii de Kenia. Más reciente encontramos la investigación de Marie-Christine Anest (1994) que aborda la zoofilia en Chipre y Creta y los trabajos de Coca, Cáceres y Valcuende (2019) y de Valcuende y Cáceres (2020).

En las últimas décadas esta temática adquiere un nuevo interés por razones científicas y sociales. Desde el punto de vista científico contemplamos una preocupación creciente por comprender "lo social" articulado con "lo ambiental", lo que implica repensar las relaciones entre los humanos y los otros seres animados e inanimados con los que nos relacionamos (Ingold 1994). La discusión sobre la frontera entre lo humano y lo animal adquiere un progresivo desarrollo en una era que algunos autores comienzan a definir como posthumanista (Wolfe 2010); esto plantea debates científicos que son, también replanteamientos de carácter moral y ético que se pueden sintetizar en dos posturas. La primera entiende que la sexualidad con animales es siempre una agresión ya que nunca puede haber consentimiento (Beirne 1997, 2001). Un segundo planteamiento considera que las relaciones sexuales con animales no implican siempre crueldad (Singer 2001). Incluso, que la zoofilia sería un modelo no antropocéntrico de relacionarse con animales (Bakke 2009). Este debate se está traduciendo en un crecimiento exponencial de normativas tendentes a proteger a otras especies no humanas y a regular las relaciones entre animales y humanos. Se abordan aspectos que hasta ahora no estaban contemplados en las distintas legislaciones; cada vez es mayor el número de normativas jurídicas que prohíben las relaciones sexuales con animales (Holoyda and Newman 2014). Las normas jurídicas tienden a contemplar a los animales, a todos los efectos, como menores sociales que hay que proteger. 


\section{Relaciones sexuales humano animales y contextos locales}

Los trabajos científicos y divulgativos que analizan las relaciones sexuales humano animales suelen comenzar con un recorrido histórico que va desde la prehistoria hasta la actualidad (Beetz 2004; Miletski 2006). Intentan demostrar que no estamos ante un comportamiento sexual nuevo ni supeditado a una cultura o a un momento histórico concreto. Nos encontramos con colecciones de ejemplos aislados que poco ayudan a entender estas conductas sexuales más allá de dejar claro que el contacto sexual humano animal es más frecuente de lo que pueda parecer. Sin embargo, si consideramos la sexualidad desde un punto de vista constructivista (Vance 1991) y partimos de su carácter simbólico (Plummer 1984) para entender una práctica sexual no basta con describirla, sino que es necesario conocer los significados que adquiere en el contexto social e histórico en el que se produce. Por eso, frente al exceso de generalizaciones creemos que hacen faltas más estudios concretos, etnografías sobre sexualidad en contextos locales (Cáceres Feria 2013). Como señala Devereux (1948) ni en todas las culturas se dan las mismas relaciones con los animales ni estas son idénticas con todas las especies. Para este autor, frente al mundo occidental que diferencia claramente a los animales de unos humanos hechos a imagen y semejanza de Dios, los mohaves de Norteamérica creen que humanos y animales originalmente no estaban diferenciados (Devereux 1948). Los mohaves y los yumas conciben las relaciones sexuales con yeguas, asnas, vacas o terneras. Ellis $(1923,79)$ cree que el bestialismo está favorecido entre los "primitivos" por una concepción de la naturaleza en la que no hay grandes barreras entre humanos y animales, y entre los campesinos por la familiaridad con sus bestias.

La sexualidad humano animal no puede analizarse como un hecho individual. Las escasas investigaciones que parten de su análisis contextual (Anest 1994; Devereux 1948; Liliequist 1991; Rydström 2003; Westermarck 2014) evidencian que existen toda una serie de pautas sociales a partir de las que se regula quiénes, cuándo, cómo y con qué tipo de animal se pueden o no mantener relaciones sexuales.

A pesar del contacto constante que los humanos mantienen con diversos animales, hay algunos con los que se puede mantener relaciones sexuales y con otros no. En Turquía, solo se puede tener relaciones sexuales con aquellos animales que no se comen, como perros y burros (Dundes, Leach, and Özkök 1970). En Grecia, sin embargo, el perro es un animal prohibido ya que se considera impuro y se teme a las enfermedades que pueda causar (Anest 1994). Entre los inuit las relaciones sexuales con perros se daban tanto en hombres como en mujeres. La sexualidad masculina con perras estaba regulada: tenía que ser al aire libre, nunca en el interior de la vivienda, y los animales debían estar en celo (Laugrand and Oosten 2002).

La sexualidad con animales se interpreta habitualmente desde una mirada masculina y casi siempre, se vincula al aislamiento y la dificultad de "acceder" a mujeres. Sin embargo, sabemos que también se da entre mujeres, aunque en distintos contextos y con características diferentes. Se suele asociar el bestialismo femenino con animales ligados al ámbito de lo doméstico como el perro (Kraft-Ebing 1894; Martín de Lucenay 1933; Matté 2008). Para Deveraux (1948) este hecho incidiría en que en muchas culturas el sexo de varones con perras se considere impensable o grotesco.

La edad será otro elemento clave a la hora de interpretar estas relaciones. Es frecuente que el sexo con animales esté ligado a los ritos de iniciación a la sexualidad y a la construcción de la masculinidad. En Suecia, desde el siglo XVII hasta principios del siglo XX, se documentan casos de 
bestialismo entre jóvenes menores de 17 años. Unos comportamientos que para Liliequist (1991) y Rydström (2003) podrían ser entendidos como una forma de experimentación sexual.

Entre los gusii de Kenia (LeVine 1959) se juzgan con cierta benevolencia las prácticas sexuales de los menores de dieciséis años con animales. Se entiende que los jóvenes están tratando de poner a prueba su capacidad sexual. En cambio, a partir de esa edad se censuran y castigan las mismas prácticas. John Money (1993) señala que entre los pueblos precolombinos del Caribe de la costa colombiana era frecuente que los jóvenes adquirieran su destreza sexual para el matrimonio mediante las relaciones sexuales con burros. No se trata de una práctica del pasado, en la actualidad en la región caribeña de La Costa en Colombia siguen dándose este tipo de contactos sexuales. A los habitantes de esta parte del país se le se les conoce como comeburras. (Delgado 1987). A temprana edad los niños comienzan a tener sus primeras experiencias sexuales con asnas. García Robayo (2010) describe en esta zona incluso prácticas de sexo de pago con estos animales. En otras áreas de Latinoamérica se han documentado también este tipo de prácticas. Así sucede en México en el que se han descrito prácticas sexuales de adolescentes con algunos animales como: burras, becerras, gallinas, pavos o chivas (Núñez Noriega 2007). Igualmente sucede en Costa Rica ${ }^{2}$ o en Honduras (Barriga et al. 2004). Sabemos que, en el Mediterráneo, la sexualidad con animales tenía un carácter colectivo y ritual. En Creta y Chipre, todavía en la década de los ochenta del siglo pasado, niños y jóvenes, entre 6 y 17 años, se iniciaban a la sexualidad manteniendo relaciones sexuales con burras, cerdas, cabras y aves hasta llegar a la edad adulta en la que se abandonaban (Anest 1994) En Anatolia, Turquía, los contactos sexuales de adolescentes con burras eran algo tolerado en las comunidades rurales (Çaya 2014; Gurkas 2008). Westermarck (2014) afirma que en Marruecos es frecuentes que niños antes de llegar a la pubertad mantengan relaciones sexuales con burros bajo la creencia que fortalece la potencia sexual y hace crecer el pene. Esas mismas prácticas en adultos se consideran ridículas y rechazables.

\section{Metodología y contexto de investigación}

El contexto territorial en el que se realiza la investigación es la zona occidental de Andalucía (España). Temporalmente se sitúa aproximadamente entre los años de 1950 a 1980, cuando la convivencia con el ganado era aún muy estrecha. La idea de realizar este análisis parte de la curiosidad que nos despierta la abundante información que de forma lateral hemos ido recopilando de distintos trabajos etnográficos, en los que los contextos de sociabilidad masculina propiciaban la conversación sobre las relaciones sexuales humano animales. Este trabajo reivindica la etnografía como una metodología adecuada también para el análisis de la sexualidad. Difícilmente, la interpretación de las relaciones sexuales humano animales hubiera sido posible sin la descripción y valoración de sus protagonistas; sin los espacios de complicidad generados en anteriores investigaciones. Es la base en la que se sostiene una de las originalidades de este trabajo: la incorporación de los testimonios directos de las personas que mantuvieron relaciones sexuales humano animales. De hecho, una parte de la información que hemos incluido en este texto proceden de los cuadernos de campo y de entrevistas realizadas en otras investigaciones realizadas en esta área.

\footnotetext{
${ }^{2}$ En el 2004 tuvimos la oportunidad de entrevistar a varios hombres en el norte de Costa Rica, en una población cercana a la frontera con Nicaragua, que relataban que era habitual que los jóvenes se iniciaran a la sexualidad con animales. Era un tema del que se hablaba entre hombres, un "secreto" entre ellos que no compartían con las mujeres. Allí, esta sexualidad se oculta a partir de la década de los ochenta del siglo pasado cuando comienzan a llegar forasteros a la comunidad que juzgan estas prácticas como depravación.
}

Revista del Laboratorio Iberoamericano para el Estudio https://doi.org/10.46661/relies.6386

Sociohistórico de las Sexualidades 
Además de la información previa con la que ya contábamos se realizaron doce entrevistas semiabiertas a hombres de entre 49 y 82 años, que directa o indirectamente formaron parte de los contextos ganaderos y territoriales aludidos, participando de los ámbitos laborales y socioculturales donde se enmarcan estas relaciones. Informantes de los que se guarda su anonimato. Con el fin de situar socio temporalmente los testimonios, las citas identifican la vinculación laboral y la edad del informante, así como el año en que se realizó la entrevista.

Distintos autores (Bernal 1988; García Sanz 1994) señalan la importancia histórica de la ganadería como fuente principal de riqueza en Andalucía destacando la complementariedad de los aprovechamientos agrícolas y ganaderos tanto en las vegas y campiñas como en las sierras (López Martínez 2005). Los sistemas de cultivo aprovechaban la ganadería como fuerza de tracción y abono en los latifundios agrícolas existentes en torno a los grandes ríos andaluces. Los animales, fundamentalmente hembras por sus posibilidades reproductivas, además de suponer una inversión en capital disponible en cualquier momento, eran clave para las tareas de labor y transporte. El ganado se alimentaba de los barbechos y restos de las cosechas (Barciela 1986) y la energía de los equinos (yeguas, mulas o burras) y vacas se empleaban en tareas tan diversas como la molienda de aceitunas, siembras, acarreo de granos, norias, etc.

En las zonas serranas menos aptas para la agricultura, la dehesa significó un modo específico de agroforestería (Nair 1993; Young 1989) que aprovechó de forma extensiva al ganado (Acosta, Díaz and Amaya 2001). Para su cuidado se contrataban a ganaderos que, con sus familias, ocupaban una vivienda y mantenían algún cerdo, aves de corral y un pequeño huerto para el autoconsumo. La sociabilidad entre los ganaderos era estrecha, así como la ocupación populosa de las hoy abandonadas dehesas serranas andaluzas. En un contexto de polaridad social donde los grandes propietarios eran absentistas y había una mayoría de población jornalera (Bernal 1988, Talego 1996) existían algunos predios donde se ubicaban pequeñas explotaciones agrarias. En ellas, el ganado mular o vacuno relacionado con el transporte o la labor se conjugaba, según las posibilidades del terreno, con la cría de especies como la cabra o la oveja, sin que faltaran aves domésticas, cerdos, etc. (García Jiménez 1997; Maestre 1968).

En los grupos familiares de jornaleros o de pequeños propietarios la especialización productiva, por género y edad, implicaba que los niños varones se ocuparan del cuidado de las aves de corral, mientras que los adolescentes se encargaban del resto de especies. Los perros se empleaban en múltiples tareas y la tenencia de una burra se convertía para los jornaleros en un medio de trabajo muy recurrente para el desarrollo de actividades autónomas.

En este contexto la relación con los animales se ajusta al modelo que Ingold (1994) describe para las sociedades de pastores, en contraposición con los pueblos cazadores-recolectores. Los animales están bajo el control del ser humano, que los cuida, los protege, los usa, pero también decide sobre la vida y la muerte.

A partir de los años cincuenta del siglo XX comienza la desaparición del ganado como elemento consustancial al latifundio andaluz. En las zonas adehesadas las crisis agrarias generan la desaparición de la mayoría de las actividades ganaderas y el abandono, despoblación y marginalización de una buena parte de las montañas andaluzas (Roux 1975). 


\section{Sexualidad con animales y ciclo vital}

Los animales formaban parte de la cotidianidad de los niños andaluces en el mundo rural. En el ámbito de la casa, en la infancia, la relación más directa era con las aves de corral, fundamentalmente gallinas y pavos. Posteriormente, a medida que se incorporan al mundo laboral y en función de su trabajo (pastor, arriero, porquero,...), se entra en contacto con animales de mayor porte: burras, cerdas, ovejas, cabras, vacas... Los niños aprenden a tratar con los diversos animales: a cuidarlos y a protegerlos, pero también cuando es necesario a castigarlos e incluso matarlos. Siguen las directrices de sus mayores, primero de las mujeres y, posteriormente, de los muchachos de más edad y de otros hombres que interactúan con las especies que están fuera de la órbita doméstica:

Los niños llevábamos la navaja en el bolsillo. Porque había veces que los pavos se embuchaban de caracoles. Y entonces, había que rajarles el buche y cosérselo, y así evitar que se murieran. Eso lo sabían hacer todos los niños. Después se quedaba el pavo dos o tres días muy triste, pero luego se espabilaba." [Pequeño Propietario, 71 años - 2004]

Los animales de corral, utilizados fundamentalmente para el autoconsumo, eran responsabilidad de las mujeres. Los niños se adiestraban con ellas en el cuidado de las aves:

Lo más cansado era cuando tenía que estar todo el tiempo vigilando a una pava para encontrar dónde ponía los huevos...Había veces que después de mucho tiempo no los encontraba. Ya cansado iba a mi madre y le decía que estaba harto. Entonces ella me decía: "tráete a la pava". Entonces yo me iba, porque coger una pava es muy fácil: tú le echas un poquillo de pan y la trincas... Se la llevaba y mi madre le echaba sal en el culo. La pava sentía la extrañeza y se iba al nidal y yo iba detrás de ella. [Pequeño Propietario, 71 años, 2005]

De forma progresiva, los niños van tomando contacto con el mundo de los hombres:

Me iba con el cabrero y nos gustaba mucho ir a los partos de las cabras, y le quitábamos los chivitos. Ordeñábamos y nos íbamos metiendo en las faenas. En el verano pastoreábamos a las vacas en la estancia" [Pequeño Propietario, 71 años, 2005]

Los menores vivían con naturalidad la vida y la muerte de los animales. En ese contacto directo aprendían de la sexualidad de los animales a partir de la que exploraban su propia sexualidad. El apareamiento de burros, yeguas, vacas, cabras, gallinas, pavos... es para ellos algo cotidiano. Unos apareamientos que, en múltiples ocasiones, no se producen de forma "natural" sino que intervienen los humanos para garantizar los cruces más apropiados o resolver problemas de cualquier índole:

A las vacas no se las follaba el toro sino el buey. Y es que el toro, aunque puede al buey, no se atrevía a pelearse con él, le tenía miedo. Entonces tuvieron que engañar al toro, pintando al buey de blanco, con cal. Al verlo pintado de cal el toro se despistó, no sabía que era el buey y se puso a pelear y le ganó, quitándole el sitio. [Pequeño propietario, 71 años, 2004]

Ahora bien, si se vivía con cierta naturalidad la presencia de los niños en la mayoría de los apareamientos, no sucede de la misma forma cuando para el acto sexual se precisaba de la mediación directa de los humanos. Había una sexualidad considerada "natural" y que podía ser vista por los niños y otra que se veía "antinatural" en la que se evitaba su presencia:

No era natural que una cabra estuviera con una vaca, o una vaca estuviera con una culebra. Las vacas se apareaban con los toros y ya está, y las cabras con las cabras...pero ahora había intención de a lo mejor echarle la yegua al caballo para que se calentara y luego echarle a la burra...y eso era forzado y ya así, yo no sé por qué, que a los niños no nos dejaban mirar. Tampoco era que nos castigaran si íbamos, pero procuraban que no estuviéramos presentes. No sé por qué, pero ya no era igual que con otros animales. [Pequeño propietario, 88 años, 2012.] 
Este tipo de apareamientos mediados por la acción humana, en especies como burros, caballos y yeguas, podía implicar la presencia de extraños al grupo doméstico. Así sucedía en algunos pueblos de las sierras andaluzas, en las que se aprovechaban los caballos sementales que el ejército proporcionaba para mejorar la cabaña ganadera. En primavera algunos ganaderos solicitaban este servicio (Salafranca 2013). Los sementales eran llevados de finca en finca para cruzarlos con las yeguas. En esos casos los padres taxativamente prohibían a los hijos menores presenciar la monta. De este modo se mostraba el recato ante los extraños (que también eran la autoridad). Se impedía que los niños viesen la intervención del denominado "mamporrero"3 que aligeraba los trámites necesarios para que los sementales eyacularan con prontitud.

A pesar de algunas restricciones impuestas por el mundo de los adultos, especialmente ante la presencia de foráneos, los niños en su cotidianidad aprenderán el significado de la sexualidad de dos formas:

-Primero, mirando a los animales a los que se imitaba jugando habitualmente con otros niños:

Mi tío tenía cabras y borregos y yo era más salvaje y les hacía las putadas a las cabras... Incluso esporádicamente jugábamos los niños desnudos a los animales, aunque no llegábamos al nivel de la penetración. [Jornalero, 70 años, 2016]

Los niños cuando eran chicos jugaban a montarse unos a otros como los animales hacían en el campo, con cinco o seis años, y nos reíamos de verlos. [Pequeño propietario, 75 años, 2015]

-Segundo, interactuando con los propios animales:

Aquella tarde fuimos a ver a tito Manolo y cuando recogimos a las cabras nos la follábamos mi primo y yo... que, iclaro!, entonces lo que hacíamos era refregar la cabra por la picha y así decíamos que nos habíamos follado a diez o doce cabras entre los dos iY no nos quitábamos ni los calzones!" [Nieto de pequeño propietario, 25 años, 1990]

Las especies más habituales, con las que los menores comienzan a interactuar sexualmente, son pequeños animales domésticos, fundamentalmente aves. A medida que los niños van creciendo se produce un contacto con animales de otro porte. El paso de un tipo de animal a otro irá definiendo las diferentes etapas de la sexualidad de los hombres. El niño dejaba de ser considerado como tal en la pubertad. El paso del mundo de la infancia al mundo de los adultos se sancionaba con la posibilidad del menor de fumar en el ámbito de trabajo en presencia del padre, no ante él. Es decir, podía llevar tabaco propio y en el descanso de cualquier actividad retirarse a fumar. Este acto simbólico marcó durante generaciones el paso entre la niñez y la edad adulta. Este periodo de transición en que el niño se sabe que fuma, pero no puede hacerlo delante de su padre, es en cierta medida un periodo considerado liminal entre la infancia y la adultez. En esta etapa se inician los escarceos con chicas, sin llegar a la penetración. También se refuerzan los contactos sexuales con animales y las prácticas onanistas vividas de forma colectiva. La virilidad debe demostrase ante los otros en toda una serie de juegos homoeróticos. Los adolescentes exploran su sexualidad y compiten por demostrar una hombría que se asocia a la posibilidad de mantener relaciones sexuales "completas". Estas prácticas son siempre furtivas y encuentran significado entre iguales; contribuyen a reforzar las vinculaciones identitarias y emocionales del grupo conformado por amigos. Unos lazos que se mantendrán, por lo general, a lo largo del tiempo. Unión y complicidad que tendrá sus consecuencias a la hora de la búsqueda del trabajo y articular estrategias de subsistencia.

${ }^{3}$ El mamporrero se encargaba de introducir el pene del caballo en la vagina de la yegua.

Revista del Laboratorio Iberoamericano para el Estudio Sociohistórico de las Sexualidades https://doi.org/10.46661/relies.6386 
Hay un hecho que sirve para diferenciar las prácticas sexuales de niños y adolescentes con animales y que está vinculado al poder de la virilidad. En determinados contextos se nos ha referido que el niño "se folla" a la pava, pero el púber "la revienta". Vemos como la "potencia" sexual se convierte en protagonista fundamental de la sexualidad masculina y define el poder a partir del cual el niño se transforma en hombre. El mostrar la posibilidad de follarse a determinados animales constituye un acto central de reafirmación de la adultez y la virilidad. Como narra en sus memorias Javier Salvago $(2007,129-130)$ originario de un pueblo de nuestra zona de estudio:

Recuerdo una noche que un compañero de la escuela nos contó que un día volvía del campo con su mula y, de repente, sintió una irresistible calentura. Se bajó del poderoso animal, se situó a su grupa, puso una piedra alta para poder llegar cómodamente a su objetivo, y una vez situado, se entregó a los bestiales placeres de la carne. Lo contaba con orgullo, como si fuera una prueba de su hombría. Cuando estaba en plena faena, nos decía, vio que se acercaba gente, pero él ya no estaba dispuesto a parar lo que había empezado. Siguió dale que te pego, sin importarle las bromas de los que estaban a su lado.

La capacidad de follarse a una burra adquiere, en el imaginario de los que van sobrepasando la etapa de la infancia, un papel central. No sólo se trataba de disfrutar sexualmente con una burra, era preciso contarlo a los otros amigos. Follarse a una burra supone un acontecimiento colectivo, ritual y que, al menos hasta los años ochenta, se ligaba al despertar sexual de los adolescentes en estos ámbitos adehesados:

Cuando íbamos al pueblo los amigos nos preguntaban si queríamos ir a follar gallinas, en una ocasión recuerdo como uno de los amigos presumía porque se había follado a una burra. [Hijo de emigrante, 50 años, $2016^{]}$

El onanismo individual y colectivo también se practicaba; eran experiencias a veces recíprocas, que más adelante, cuando se empieza a tener relaciones sexuales con mujeres, corren el riesgo de ser interpretadas en clave de "homosexualidad". De hecho, sobre estas prácticas caerá un incómodo silencio cuando alguien del grupo las recuerde en la edad adulta.

La interpretación de la sexualidad tenía un carácter marcadamente diferente para los muchachos y las muchachas. En el caso de los primeros la sexualidad es un elemento central en el proceso de socialización. Para las mujeres la sexualidad correcta se interpreta en clave reproductiva Así, las chicas que salían con muchos chicos eran deseables sólo para el sexo, no para conformar una familia. Juana explicitaba esta situación a los dieciséis años:

$Y$ es que ya he tenido dos novios y el tercero tiene que ser el último...porque es que, si no, todo el mundo va a decir que soy una puta y eso no puede ser... ya el siguiente es con el que me quedo. [Jornalera, 16 años, 1989]

El noviazgo y el matrimonio marcan teóricamente el final de las relaciones sexuales con animales. En algunos casos continúan, pero la vivencia pasará a ser individual, no colectiva. La valoración de las prácticas sexuales con los animales, normalizada en determinadas edades, comienza a verse extraña en la edad adulta. De la misma forma ocurre en relación a las masturbaciones colectivas:

Si tú ya dejabas de tener cabras y te incorporaba a otros trabajos con 16 ó 17 años y en la adolescencia tenías relaciones con mujeres ya no te vas a follar una burra. [Jornalero, 60 años, 2011].

La separación entre la esfera doméstica y los ámbitos laborales al margen de la casa se articulan en nuevos contextos "de solidaridad" masculina vinculados a las prácticas sexuales de pago. EI matrimonio no significa en muchos casos que no haya prácticas sexuales extramatrimoniales. Ahora también algunos hombres vivirán su sexualidad fuera del hogar a partir del sexo de pago, en las casas de prostitución. 
La sexualidad con prostitutas se vincula precisamente a los ámbitos productivos. El "irse de putas" es un acto que se hace en grupo con los amigos, que suelen ser compañeros de trabajo. No es normalmente una acción individual, como tampoco lo es tomar alcohol; uno y otro elemento están estrechamente vinculados y ambos forman parte de los contextos y de las formas de sociabilidad masculina. Una sociabilidad que se reafirma en el secreto de la sexualidad propia de los hombres y que nunca se explicita ante las mujeres que forman parte del ámbito "reproductivo". El deseo, el placer, la liberación de la cotidianeidad, se conciben ahora desde el consumo accesible al sexo de pago:

(El sexo con animales) no era motivo de burla...porque en el entorno en el que se hablaba era un entorno muy leal. Esa gente no hacía estas cosas con cualquiera. Ellos lo compartían con sus colegas, en entorno con complicidad...eso era como cuando nos hacíamos pajas juntos y a ver quién se corría antes. Y eran espacios de complicidad como cuando luego se va de putas. Igual, se mantiene la complicidad. [Trabajador, 65 años, 2016].

\section{La normatividad de las prácticas sexuales con animales y las desviaciones de la norma}

Como hemos visto a través de los testimonios recopilados la sexualidad con animales en los contextos agrarios andaluces estaba reglada socialmente. Ni en todas las etapas de la vida se consideraban adecuadas ni todas las especies tenían la misma significación. Conviene destacar algunos de estos aspectos:

1. La mayor parte de los contactos sexuales con animales reproducen la lógica de la sexualidad heteronormativa. De hecho, las prácticas sexuales de los varones se realizan fundamentalmente con animales hembras. Solamente uno de los informantes alude a prácticas sexuales con un burro macho y lo justifica porque su padre no disponía de hembras. A su vez, destaca su virilidad ante la dificultad que entraña copular con un macho:

Yo me he cogido un borrico cuando era chico... ique una burra es más ligera! Nosotros de chico hemos tenido borrico [...] Yo iba a trabajar al río montado en un borrico, y estás to el camino armado. iVas con las patas abiertas, y te vas frotando! iEso es horroroso! ilncluso montado en un borrico! iTe amarrabas al atarraje del borrico y te lo follabas y se acabó! [Jornalero, 76 años, 2016]

2. En función de la etapa del ciclo vital, la sexualidad con animales y los propios animales tienen una distinta consideración Las aves de corral están asociadas al mundo de la infancia. Y como veíamos anteriormente, uno de los hitos en el tránsito hacia la edad adulta es la sexualidad con pavos, "reventar a las pavas". Pero el paso definitivo hacia la adultez es buscar animales de mayor porte que se encuentran en el ámbito laboral. La capacidad de follarse a una burra constituye una experiencia fundamental en la reafirmación de la sexualidad frente al grupo de iguales.

3. Las relaciones sexuales con animales durante una etapa de la vida deben tener un carácter colectivo. Esto no implica que estas prácticas se realicen siempre en grupo, pero, en todo caso, deben ser sabidas y conocidas por la pandilla de amigos, aunque nunca fuera de la misma. Igualmente sucederá en la edad adulta cuando la sexualidad se vincula con las casas de 
prostitución. En esas prácticas colectivas y/o socializadas las relaciones con los animales son puramente instrumentales, no es habitual mostrar afecto hacia ellos.

4. La elección de los animales con los que se va a mantener relaciones sexuales está marcada por varios factores. El primero, con las desiguales posibilidades de acceso a unos y otros animales:

Yo no me relacionaba con cabras porque estaba en el pueblo. (...) Pero amigos porqueros lo hacían con cochinas, y el cabrero con las cabras y el pastor con las ovejas... y con burras, que eran más cómodas... Tú hablas con gente y en función con el sector que trabajaba así se enrollaba o por lo menos lo intentaban." [Obrero, 66 años, 2015]

Algunos de los del pueblo nos íbamos con las burras que siempre estaban en las cañadas". [Obrero, 67 años, 2011].

El segundo elemento está relacionado con las propias características fisiológicas de cada especie y su actitud ante la penetración que las hacen más o menos apropiadas a la hora de mantener relaciones sexuales. A nivel discursivo se van definiendo las características y cualidades de algunos de estos animales:

Por lo visto la vagina de las cochinas es muy peculiar porque el pene del cochino ya has visto como es: una especie de broca. [Jornalero, 60 años, 2015]

La vaca es respingona, la burrita es buena y quieta y la mejor la oveja que está bien templá. [Arrendatario, 52 años, 1987]

Si bien estos factores son importantes, en cuanto que propician o no la posibilidad de mantener relaciones sexuales con animales, no son determinantes. De hecho, hay animales con los que se tiene un contacto habitual y con los que en este contexto no se mantienen relaciones sexuales. Sin duda el caso más emblemático son los perros. Los perros no están en el imaginario sexual y no es una relación socialmente asumida. El perro en cierta medida es un animal con el que tener sexo es un tabú para las personas que sí han mantenido sexo con otros animales. Con los perros se podía como mucho "refregarse" pero no realizar el coito:

Con perros te podías refregar, pero no penetrarlo [Jornalero, 60 años, 2013]

Con trece o catorce años cuando se te acercaba una perra le acariciabas, pero no lo intentabas... porque te daba asco. Aunque te arrimases a la perra, no la penetrabas, con los perros nos daba asco. [Jornalero, 45 años, 2000]

Lo más asqueroso era los perros, a los perros no se le tocaba... a lo mejor porque tú lo veías follando en las calles y llenos de baba. Yo tengo una anécdota que en un descanso mientras trabajaba había un perro pegado a una perra y cuando estaba viéndolo me estaba comiendo un bocadillo de morcilla...y me tiré veinte años aborreciendo la morcilla del asco que me dio. ¡Que veíamos a perros pegados y les dábamos garrotazos!... iHay que ver lo que éramos! [Obrero, 65 años, 2016]

5. De manera general los contactos sexuales con animales comienzan a ser vistos como anómalos a partir del proceso de noviazgo y el posterior matrimonio. Sin embargo, hay causas que justifican la continuidad de esta sexualidad; fundamentalmente es el caso de los hombres solteros que por su trabajo están largos periodos de tiempo entre animales. La proximidad a los animales, vivir en el campo y fundamentalmente la soltería podían prolongar este tipo de 
prácticas, incluso el desarrollar otro tipo de sentimientos como el afecto, que no aparece en las prácticas colectivas; en este caso se habla de apego:

Le das el caramelo a la cabrita, que está "apegaita" a mí. iY es que es más cariñosa y más buena!". [Jornalero, 50 años, 2001].

Sin embargo, aquí se define uno de los límites de la permisividad y de lo que se considera o no como "normal":

Éste iba del pueblo al río todas las tardes. Que ya nosotros moceábamos, pero este se iba a la burra del lechero y allí se tiraba después de comer hasta que volvía y llegaba a jugar a las cartas con nosotros. Una tarde llegó con pena, medio llorando: dijo que no estaba, que la burra se había muerto... y estuvo una temporada entristecido. [Jornalero, 40 años, 1999]

Existen situaciones que dificultan la posibilidad de adecuarse a la heteronormatividad en pareja, que está en la base de la concepción de familia tradicional. Si bien estas circunstancias pueden llegar a ser comprendidas pasan a ser valoradas como "desviación" social. El siguiente testimonio es especialmente interesante precisamente a la hora de aproximarnos a lo que está permitido y aquello que se encuentra en una situación liminal. El informante que durante su infancia formó parte de los contextos agrarios andaluces, fue uno de los muchos emigrantes que, en los años sesenta, tuvo que irse a trabajar a la ciudad, regresando nuevamente al área rural. Estamos en una fase de transición que pone de manifiesto las contradicciones entre los valores oficiales que se imponen en la sociedad industrial y la costumbre:

Conocí a una familia en la sierra y el crío que todavía me acuerdo del nombre, tenía una novia que era su cabra. Y la familia le permitió que él se acostara con su cabra en la cama. Eso fue un choque para mí. $Y$ yo he sido un hombre abierto con mis contradicciones... y yo pensaba que había que intervenir para ayudarle... ipero qué coño soy yo! Sus padres eran pastores desde que se recordaba y no sabían ni hablar. El padre y los dos hijos siempre habían dormido en el campo, mientras que las hijas vivían en el pueblo con la madre. Y los chavales tenían relaciones con las cabras. Los fines de semana cuando eran más grandes podian escaparse para echar un polvete donde podían (prostitutas). Pero el chico aquel que estaba allíque tenía una discapacidad ese tuvo una relación afectiva con la cabra permanente y estable. [Obrero, 68 años, 2016]

Como hemos podido ver, a lo largo de este trabajo, no podemos entender la sexualidad con animales como una excepcionalidad, una perversión o algo marginal fuera de la norma. Hemos intentado mostrar precisamente cómo a partir de la heteronormatividad pueden entenderse estas prácticas sexuales, vinculadas a la construcción de la masculinidad, basada en los fuertes lazos generados entre hombres y protegida por "los secretos". Unos hombres que aprenden desde niños la significación social del pene como símbolo de poder.

Estas prácticas, aunque habituales en las poblaciones que hemos estudiado, no eran compartidas por todos los hombres. Y, ciertamente desde hace décadas está forma de entender la sexualidad está transformándose. Hoy las relaciones con animales son objeto de burla y eso hace que cada vez sea más difícil encontrar testimonios de un tipo prácticas que fue generalizada y que pudo mantenerse en una realidad relativamente escondida y protegida por los grupos de hombres. 


\section{Conclusiones}

Las críticas a las visiones patológicas de las relaciones sexuales humano animal, aunque han sido minoritarias, no son nuevas (Pomeroy 1977); como tampoco lo son las que inciden en algunos de los problemas metodológicos de este enfoque. Los datos utilizados en muchos trabajos proceden mayoritariamente de informes criminológicos que incluyen individuos violentos con trastornos psiquiátricos (Beetz 2004). En su famoso informe, Kinsey (1948) puso en evidencia la visión antropocéntrica y los prejuicios morales en las aproximaciones de la biología a las relaciones sexuales interespecies, negadas o invisibilizadas ${ }^{4}$ durante mucho tiempo. Si esto ha sucedido con otras especies no es extraño que a la sexualidad humano animal solo se le haya prestado una especial atención como "desviación" o "patología" e, incluso, como delito.

La psiquiatría y la medicina coparon buena parte de unas investigaciones que entienden la sexualidad humana como un hecho puramente biológico, olvidando el carácter marcadamente social de unas prácticas que no pueden ser entendidas al margen de sus significaciones. La centralidad que se le otorgan a las prácticas, obviando su carácter contextual, ha provocado que muchos estudios históricos recopilen datos fragmentarios y descontextualizados que pretenden poner en evidencia su carácter universal. Sin embargo, una misma práctica en distintas sociedades y momentos históricos puede encerrar significaciones marcadamente diversas.

Los animales juegan un papel central en el aprendizaje de la sexualidad de los niños y adolescentes campesinos. Los niños explorarán su sexualidad mirando a los animales, interaccionando con ellos y descubriendo el cuerpo con otros niños. Aprenden el valor de su cuerpo y de su pene que debe ser mostrado y explorado con el grupo de iguales. Competirán por ver "quién la tiene más larga o quién mea más lejos" (Sabuco y Valcuende 2003). En el aprendizaje compartido de la sexualidad rivalizan por demostrar quién es capaz de follarse, primero, a las aves de corral y, posteriormente, a mamíferos como burras, cerdas, ovejas y cabras. Los adolescentes varones asimilan que ellos son los destinados al poder público; un poder que se ejercerá también en unas relaciones sexuales reinterpretadas en clave masculina. Este proceso de aprendizaje de la sexualidad tiende a reforzar el papel de "las pandillas", "las quintas", los grupos de pares (Núñez Noriega 2007). Grupos de la misma generación que compartirán primero los juegos y posteriormente los ámbitos de sociabilidad y trabajo (Cantero 1996).

En Andalucía, como en todo el ámbito mediterráneo, ha existido una fuerte segregación de los espacios femenino y masculino (Anest 1994; Gilmore 1990). El mundo de los hombres se vinculaba a los contextos laborales fuera de la casa, a los espacios públicos de sociabilidad como las tabernas. El universo femenino se vincula al denominado ámbito "reproductivo" y al mantenimiento de las redes familiares. Lo femenino y lo masculino son mundos que conviven en paralelo, dos formas de sociabilidad que marcarán también dos modos de entender la sexualidad, percibidos como jerárquicamente complementarios (Valcuende, 2004). Esta fuerte separación permitirá una serie de prácticas consentidas y protegidas desde la solidaridad masculina que se refuerza a través del "secreto" de una sexualidad no potencialmente reproductiva. Es en ese contexto en el que la sexualidad con animales adquiere significación.

La observación y la interacción entre los niños y adolescentes, y entre estos y los animales reforzaban los ámbitos de sociabilidad masculina. Los niños interactuaban con los animales, jugaban a "ser animales" y también jugaban a "ser hombres". La masculinidad se vincula de forma directa a la posibilidad de mantener relaciones sexuales con penetración. Un modelo en negativo que se

\footnotetext{
${ }^{4}$ Nos parece llamativo que, como señala Maple (cited by Money, 1993, 99), no exista un término equivalente a zoofilia para referirse a relaciones sexuales entre dos especies no humanas.
}

Revista del Laboratorio Iberoamericano para el Estudio https://doi.org/10.46661/relies.6386

Sociohistórico de las Sexualidades 
construye en contraste con "los otros": las mujeres, los menos hombres, los niños (Gilmore 1990; Kimmel 1997) y también los animales.

La conformación de la pareja refuerza la dicotomía entre la sexualidad reproductiva, que está en la base de la familia, y una sexualidad vivida al margen de la misma. Es precisamente el acceso a las relaciones sexuales en pareja heterosexual las que a nivel discursivo ponían freno a las relaciones sexuales humano animales. Las relaciones sexuales con animales de determinados grupos en el mundo rural no pueden ser interpretadas necesariamente como una desviación y mucho menos como una patología. Estas prácticas formaban parte de la heternormatividad e, incluso, reproducían algunas de sus reglas (preferencia por las relaciones sexuales con hembras). A pesar de que algunos individuos establecían ocasionalmente vínculos emocionales con los animales, estas relaciones no están en la base de la construcción de una identidad sexual singular, zoosexualidad, como se plantea desde determinados ámbitos de investigación. Pero tampoco son la mera la expresión de un deseo sexual desbordado que busca exclusivamente la satisfacción individual, bestialismo, ya que tenían toda una serie de reglas. Las prácticas sexuales humano animales no se entienden exclusivamente desde las relaciones que establecen los hombres con los animales sino, sobre todo, a partir de los vínculos que establecían los hombres entre ellos y con las mujeres. 


\section{Referencias}

Acosta Naranjo, Rufino, Antonio Luis Díaz Aguilar, and Santiago Amaya Corchuelo. (2001). Memoria de La Tierra, Campos de La Memoria: Los Agroecosisternas Tradicionales de Tentudía. Monesterio, Mérida: Centro de Desarrollo Comarcal de Tentudía.

Aggrawal, Anil. (2011). "A New Classification of Zoophilia." Journal of Forensic and Legal Medicine 18 (2): 73-78.

Alvarez, William A. and Jack. P. Freinhar. (1991). "A Prevalence Study of Bestiality (Zoophilia) in Psychiatric in-Patients, Medical in-Patients, and Psychiatric Staff." International Journal of Psychosomatics 38 (1-4): 45-47.

American Psychiatric Association. (2013). Diagnostic and Statistical Manual of Mental Disorders (DSM- $5^{\circledR}$ ); Washington, DC, USA: American Psychiatric Publishing.

Amodio, Emanuele. (2012). "El Detestable Pecado Nefando. Diversidad Sexual y Control Inquisitorial en Venezuela Durante el Siglo XVIII." Nuevo Mundo Mundos Nuevos.

Anest, Marie-Christine. (1994). Zoophilie, Homosexualité, Rites de Passage et Initiation Masculine Dans la Grèce Contemporaine Paris: Editions L'Harmattan.

Ascione, Frank R. (2005). "Bestiality: Petting, Humane Rape, Sexual Assault, and the Enigma of Sexual Interactions between Humans and Non-Human Animals." Anthrozoos-Journal of the International Society for Anthrozoology 18: 120-129.

Bakke, Monika. (2009). "The Predicament of Zoopleasures: Human-Nonhuman Libidinal Relations." In Animal Encounters, edited by Tom Tyler and Manuela S. Rossini, 221-242. Leiden: Brill.

Barciela López, Carlos. (1986). "Los Costes del Franquismo en el Sector Agrario: la Ruptura del Proceso de Transformaciones. Parte 2, Introducción." In Historia Agraria de la España Contemporánea (1900-1960), T. 3, edited by Ramón Gabarrou, Carlos Barciela López and José Jiménez Blanco, 383-454. Barcelona: Crítica.

Barriga, Patricio, Rudy C. Rosales de Molinero, and Jorge A. Fernández V. (2006). "Comportamientos Sexuales de Adolescentes y Jóvenes Adultos en Comunidades de Honduras." Revista Médica Hondureña 74 (1): 4-18.

Bazant, Mílada. (2002). Bestialismo: El delito Nefando, 1800-1856. México, DF: Fondo de Cultura Económica.

Beetz, Andrea M. (2002). Love, Violence, and Sexuality in Relationships between Humans and Animals. Aachen, Germany: Shaker.

Beetz, Andrea M. (2004). "Bestiality/Zoophilia: A Scarcely Investigated Phenomenon between Crime, Paraphilia, and Love." Journal of Forensic Psychology Practice 4 (2): 1-36.

Beidelman, Thomas O. (1961). "Kaguru Justice and the Concept of Legal Fictions." Journal of African Law 5 (1): 5-20.

Beirne, Piers. (2001). "Peter Singer's Heavy Petting and the Politics of Animal Sexual Assault." Critical Criminology 10 (1): 43-55.

Beirne, Piers. (1997). "Rethinking Bestiality: Towards a Concept of Interspecies Sexual Assault." Theoretical Criminology 1 (3): 317-340.

Bernal Rodríguez, Antonio Miguel. (1988). Economía e Historia de los Latifundios. Madrid: Espasa Calpe. 
Cáceres Feria, Rafael. (2013). "Diversidad Sexual: Contextos Locales, Discursos Globales." In Estudios Sobre Diversidad Sexual en Iberoamérica. Sexualidades y Culturas, edited by Valcuende, José María, María J. Marco Macarro and David Alarcón Rubio, 15-25. Sevilla: Aconcagua.

Coca-Pérez, Agustín; Cáceres-Feria, Rafael y Valcuende del Río, José María (2019). Human-animal sexual relations and the construction of masculinity in livestock farming contexts: The case of Andalusia (Spain). Sexualities. 2019;22(7-8):1017-1034. doi:10.1177/1363460718790886

Connell, R; Messerschmidt, J. W. Traducción de: Stéfano Barbero, M., \& Morcillo, S. (2021). Masculinidad hegemónica. Repensando el concepto. RELIES: Revista Del Laboratorio Iberoamericano Para El Estudio Sociohistórico De Las Sexualidades, (6), 32-62. https://doi.org/10.46661/relies.6364

Cantero, Pedro Antonio. (1996). "La Quinta De Galaroza." Demófilo: Revista de Cultura Tradicional (19): 15-36.

Çaya, Sinan. (2014". "Violence in Rural Regions: The Case of ModernTurkey." Procedia-Social and Behavioral Sciences 114: 721-726.

Cerrone, Gerald H. (1991). "Zoophilia in a Rural Population: Two Case Studies." Journal of Rural Community Psychology 12 (1): 29-39.

Chaplin, James H. (1963). "A Report on Sexual Behavior: Six Case Histories from Northern Rhodesia." Advances in Sex Research 1: 13-26.

Davis, Daniel L. and Richard G. Whitten. (1987). "The Cross-Cultural Study of Human Sexuality." Annual Review of Anthropology 16: 69-98.

Dekkers, Midas. (1994). Dearest Pet: On Bestiality New York: Verso Books.

Delaney, Carol. (1991). The Seed and the Soil: Gender and Cosmology in Turkish Village Society. Berkeley: University of California Press.

Delgado, Ramiro. (1987). "Cotidianidad y Fiesta en el Municipio de Talaigua: Relatos de la Gente." Boletín Cultural y Bibliográfico 24 (12): 29-49.

Devereux, George. (1948). "Mohave Zoophilia." Samiksha: Journal of the Indian Psychoanalytical Society 2 (3): 227-245.

Duffield, Gary, Angela Hassiotis, and Eileen Vizard. (1998). "Zoophilia in Young Sexual Abusers." The Journal of Forensic Psychiatry 9 (2): 294-304.

Dundes, Alan, Jerry W. Leach, and Bora Özkök. (1970). "The Strategy of Turkish Boys Verbal Dueling Rhymes." The Journal of American Folklore 83 (329): 325-349.

Durkin, Keith, Craig J. Forsyth, and James F. Quinn. (2006). "Pathological Internet Communities: A New Direction for Sexual Deviance Research in a Post Modern Era." Sociological Spectrum 26 (6): 595-606.

Earls, Christopher M. and Martin L. Lalumière. (2009). "A Case Study of Preferential Bestiality." Archives of Sexual Behavior 38 (4): 605-609.

Ellis, Havelock. (1923). Studies in the Psychology of Sex.Erotic: Symbolism the Mechanism of the Detumescence the Psychic State in Pregnancy. Philadelphia: F. A. Davis Company.

Evans-Pritchard, Edward E. (1956). Nuer Religion. Oxford: Oxford University Press.

Flynn, Clifton P. (1999). "Animal Abuse in Childhood and Later Support for Interpersonal Violence in Families." Society \& Animals 7 (2): 161-172. 
Foucault, Michel. (1976). Histoire de la Sexualité. 1, La Volonté De Savoir. Paris: Gallimard.

Foucault, Michel. (1975). Surveiller et Punir: Naissance de la Prison. Paris: Gallimard.

García Jiménez, Guillermo. (1997). Lamento Campesino. Un Siglo Crucial en la Historia de Alcalá de los Gazules (1860-1960). Cádiz: Ediciones Publicaciones del Sur.

García Robayo, Margarita. (2010). Burdel de Burras. accessed, December 5, 2016, https://cronicasperiodisticas.wordpress.com/2010/05/13/burdel-de-burras/ (fecha de consulta 10/12/2021).

García Sanz, Ángel. (1994). "La Ganadería Española entre 1750-1865: Los Efectos de la Reforma Agraria Liberal." Agricultura y Sociedad (72): 81-120.

Gilmore, David D. (1990). Manhood in the Making: Cultural Concepts of Masculinity. New Haven \& London: Yale University Press.

Gurkas, Hakki. (2008). Nasreddin Hodja and the Aksehir Festival: Invention of a Festive Tradition and Transfigurations of a Trickster, from Bukhara to Brussels. West Lafayette, Indiana: Purdue University.

Hensley, Christopher, Suzanne E. Tallichet, and Stephen D. Singer. (2006). "Exploring the Possible Link between Childhood and Adolescent Bestiality and Interpersonal Violence." Journal of Interpersonal Violence 21 (7): 910-923.

Holoyda, Brian and William Newman. (2014). "Zoophilia and the Law: Legal Responses to a Rare Paraphilia." Journal of the American Academy of Psychiatry and the Law 42 (4): 412-420.

Ingold, Tim. (1994a). "From Trust to Domination: An Alternative History of Human-Animal Relations." In Animals and Human Society: Changing Perspectives, edited by Aubrey Manning and James Serpell, 1-22. London: Routledge.

Ingold, Tim. (1994b). "Introduction." In What is an Animal?, edited by Tim Ingod, 1-16. London \& New York: Routledge.

Jenkins, Robert E. and Alexander R. Thomas. (2004). Deviance Online: Portrayals of Bestiality on the Internet. Oneonta, NY: Center for Social Science Research.

Kavanaugh, Philip R. and R. J. Maratea. (2016). "Identity, Resistance and Moderation in an Online Community of Zoosexuals." Sexualities 19 (1-2): 3-24.

Kimmel, Michael S. (1997). "Masculinity as Homophobia: Fear, Shame and Silence in the Construction of Gender Identity." In Toward a New Psychology of Gender, edited by Mary M. Gergen and Sara N. Davis, 223-242. Florence, KY, US: Taylor \& Francis/Routledge.

Kinsey, Alfred Charles, Wardell Baxter Pomeroy, and Clyde Eugene Martin. (1948). Sexual Behavior in the Human Male. Philadelphia: W. B. Saunders.

Krafft-Ebing, Richard von. (1894). Psychopathia Sexualis. New York: Rebman.

Laugrand, Frédéric and Jarich Oosten. (2002). "Canicide and Healing. the Position of the Dog in the Inuit Cultures of the Canadian Arctic." Anthropos 97: 89-105.

LeVine, Robert A. (1959). "Gusii Sex Offenses: A Study in Social Control." American Anthropologist 61 (6): $965-990$.

Liliequist, Jonas. (1991). "Peasants Against Nature: Crossing the Boundaries between Man and Animal in Seventeenth-and Eighteenth-Century Sweden." Journal of the History of Sexuality 1 (3): 393-423. 
López Martínez, Antonio Luis. (2005). "Una Élite Rural. Los Grandes Ganaderos Andaluces, Siglos XIV-XX." Hispania 65 (221): 1023-1042.

Maestre, Juan. (1968). Hombre, Tierra y Dependencia en el Campo de Gibraltar: Un Estudio de Campo en una Zona Subdesarrollada. Madrid: Ciencia Nueva.

Malinowski, Bronislaw. (1975). La Vida Sexual de los Salvajes del Noroeste de la Melanesia. Madrid: Morata.

Maratea, Richard J. (2011). "Screwing the Pooch: Legitimizing Accounts in a Zoophilia On-Line Community." Deviant Behavior 32 (10): 918-943.

Martín de Lucenay, Ángel. (1933). Bestialismo. Madrid: Fénix.

Matté, Aline Karen. (2008). "Prazeres Velados e Silêncios Suspirados: Sexualidade e Contravenções na Região Colonial Italiana: 1920-1950."Pontifícia Universidade Católica do Rio Grande do Sul.

McIntosh, Mary. (1968). "The Homosexual Role." Social Problems 16 (2): 182-192.

Miletski, Hani. (2001). "Zoophilia: Implications for Therapy." Journal of Sex Education and Therapy 26 (2): 85-89.

Miletski, Hani.(2006). "Updates of Clinical and Educational Sexology: Introduction to Bestiality and Zoophilia " Contemporary Sexuality 40 (12): 8-13.

Miletski, Hani. (2017). "Zoophilia: Another Sexual Orientation?" Archives of Sexual Behavior 46 (1): 39-42.

Money, John. (1993). Lovemaps: Clinical Concepts of Sexual/Erotic Health and Pathology, Paraphilia, and Gender Transposition of Childhood, Adolescence, and Maturity. New York: Irvington Publishers.

Nair, P.K. Ramachandran. (1993). An Introduction to Agroforestry. Dordrecht, Netherlands: Kluwer Academic.

Navarro, John C. and Richard Tewksbury. (2015). "Bestiality: An Overview and Analytic Discussion." Sociology Compass 9 (10): 864-875.

Núñez Noriega, Guillermo. (2007). Masculinidad e Intimidad: Identidad, Sexualidad y Sida. México, DF: Universidad Autónoma de México.

Peretti, Peter O. and Maurice Rowan. (1982). "Variables Associated with Male and Female Chronic Zoophilia." Social Behavior and Personality: An International Journal 10 (1): 83-87.

Plummer, Ken. (1984). "Sexual Diversity: A Sociological Perspective." In The Psychology of Sexual Diversity, edited by Kevin Howells, 219-253. London: Blackwell.

Pomeroy, Wardell B. (1977). "Sexual Contact with Animals." Penthouse Forum (October).

Roux, Bernard. (1975). Crisis Agraria en la Sierra Andaluza. Vol. 3. Sevilla: Universidad de Sevilla.

Rubin, Gayle S. (1984). "Thinking Sex: Notes for a Radical Theory of the Politics of Sexuality." In Pleasure and Danger: Exploring Female Sexuality, edited by Carole S. Vance. Boston: Routledge and Kegan Paul.

Rydström, Jens. (2003). Sinners and Citizens: Bestiality and Homosexuality in Sweden, 1880-1950. Chicago: University Chicago Press.

Sabuco, Assumpta and José María Valcuende. (2003). "La homosexualidad como Imagen Hiperbólica la Masculinidad." In Hombres: La Construcción Cultural de las Masculinidades, edited by José María Valcuende and Juan Blanco, 135-155. Madrid: Talasa. 
Salafranca Álvarez, Juan Ignacio. (2013). "La Cría Caballar, Aportación del Ejército a la Sociedad Civil." Revista de Historia Militar LVII (II): 179-196.

Salisbury, Joyce E. (1991). "Bestiality in the Middle Ages." In Sex in the Middle Ages: A Book of Essays, edited by Joyce E. Salisbury, 173-186. London: Garland.

Salvago, Javier. (2007). Memorias de un Antihéroe. Sevilla: Renacimiento.

Singer, Peter. (2001)."Heavy Petting." Nerve, accessed, October 7, 2016, https://www.utilitarian.net/singer/by/2001----.htm.

Talego, Félix. (1996). Entre el Trabajo y Los Subsidios de Desempleo: Los Jornaleros de Lebrija. Lebrija, Sevilla: Hermandad de los Santos.

Vance, Carole S. (1991). "Anthropology Rediscovers Sexuality: A Theoretical Comment." Social Science \& Medicine 33 (8): 875-884.

Valcuende del Río, José María. (2003). A modo de introducción. Una aproximación a las masculinidades. Hombres: la construcción cultural de las masculinidades. Sevilla: Talasa.

Valcuende del Río, José María (2004). Cuerpos, Géneros y Sexualidades: Representaciones y prácticas sociales. Revista crítica jurídica, 23, 150-173.

Valcuende del Río, José María y Cáceres-Feria, Rafael. (2020). Analysis of Human-Animal Sexual Interactions. Animals, 10, 1780. https://doi.org/10.3390/ani10101780

Vega Umbasia, Leonardo Alberto. (1994). Pecado y Delito en la Colonia: La Bestialidad como una Forma de Contravención Sexual (1740-1808). Bogotá: Instituto Colombiano de la Cultura Hispánica, accessed, November 10, 2016, http://catalog.hathitrust.org/Record/003158352

Westermarck, Edward. (2014). Ritual and Belief in Morocco Vol. II. Abington: Routledge.

Williams, Colin J. and Martin S. Weinberg. (2003). "Zoophilia in Men: A Study of Sexual Interest in Animals." Archives of Sexual Behavior 32 (6): 523-535.

Williams, Thomas R. (1966). "Cultural Structuring of Tactile Experience in a Borneo Society." American Anthropologist 68 (1): 27-39.

Wolfe, Cary. (2010). What is Posthumanism? Minneapolis: University of Minnesota Press.

Young, Anthony. 1989. Agroforestry for Soil Conservation. Wallingford, UK: CAB international. 